\title{
Quantitative brain MR imaging in amyotrophic lateral sclerosis
}

\author{
Jiř́ Keller · Josef Vymazal · Petr Ridzoň • \\ Robert Rusina • Petr Kulišsták • Hana Malíková • \\ Aaron M. Rulseh • Otakar Keller • Robert Jech
}

Received: 8 July 2010 / Revised: 26 November 2010 / Accepted: 29 November 2010 / Published online: 15 March 2011

(C) The Author(s) 2011. This article is published with open access at Springerlink.com

\begin{abstract}
Object To evaluate the potential of quantitative MR techniques [voxel-based morphometry (VBM), T2-relaxometry, mean diffusivity (MD), fractional anisotropy (FA)] in the diagnostics of amyotrophic lateral sclerosis (ALS).

Materials and methods Thirty-three ALS patients and thirty age- and sex-matched healthy volunteers were included in the cross-sectional study. T1WI, T2WI and T2 relaxometry sequences were performed at 1.5T. DWI was performed in a subgroup of 12 patients. Disease severity was estimated with the ALS Functional Rating Scale (ALS-FRS).

Results We detected decreased T2 relaxation rate (R2) in the frontal white matter (FWM) (left and right $P<0.005$ ) and caudate nucleus (left $P<0.005$ ) in ALS patients. R2 in the FWM correlated with age in patients and controls. A correlation $(P<0.01$, cluster-level corrected) between

Preliminary data were presented on ESMRMB meeting in Antalya in 2009 as a lecture (abstract: Keller J, Vymazal J, Jech R, Ridzoň P, Rusina R, Keller O: Quantitative MR Imaging in amyotrophic lateral sclerosis. Magma Magn Reson Mater Phys Biol Med, 2009, 22, Suppl. 1, p.131).
\end{abstract}

\section{J. Keller}

Third Faculty of Medicine, Charles University in Prague, Prague, Czech Republic

J. Keller · J. Vymazal (凶) · H. Malíková · A. M. Rulseh Department of Radiology, Na Homolce Hospital,

15030 Prague 5, Czech Republic

e-mail: josef.vymazal@homolka.cz

J. Vymazal · R. Jech

Department of Neurology and Center of Clinical Neuroscience,

First Faculty of Medicine, Charles University in Prague,

Prague, Czech Republic

P. Ridzoň · R. Rusina · P. Kulišt’ák · O. Keller

Department of Neurology, Thomayer Teaching Hospital,

Prague, Czech Republic atrophy in the corona radiata and the limb ALS-FRS subset was found, as well as a difference between patients and controls in this area. No correlation between FA/MD and ALS-FRS was observed in the T2 hyperintense region of the posterior limb of the internal capsule (PLIC), or in the site of atrophy detected by VBM. No R2 or PD changes in the PLIC were detected. TBSS revealed decreased FA in the corona radiata and callosal body.

Conclusions Decreased R2 in the left caudate and bilateral FWM may help in the diagnostic process and disqualifies these regions as internal controls in ALS studies. The PLIC is not a reliable diagnostic marker of ALS.

Keywords Amyotrophic lateral sclerosis - T2 relaxation time - Fractional anisotropy - Voxel-based morphometry . ALS-FRS $\cdot$ Caudate $\cdot$ Frontal white matter $\cdot$ R2

\section{Introduction}

Amyotrophic lateral sclerosis (ALS) is the most common motor neuron disease (MND) - a group of neurological disorders which selectively affect upper and lower motor neurons. This disease carries a fatal prognosis, its aetiology is still little understood, and no specific treatment yet exists. Traditionally, ALS has been described as a neurodegenerative disorder primarily affecting motor neurons in the anterior horns of the spinal cord, bulbar nuclei and cerebral motor cortex [1-3], with secondary degeneration of the emerging tracts of both the central and peripheral nervous systems [4]. Clinically, two forms of ALS may be distinguished according to the site of dominant involvement: a limb-onset and a bulbar-onset form. During the span of the disease, both forms include progressive muscle weakness, including the muscles needed for speech, swallowing and breathing. 
Based on recent studies [5,6], the perspective regarding ALS has evolved to include the broader involvement of central nervous system regions beyond the motor system. Frontal lobe involvement has been detected in ALS, and together with the cognitive impairment of approximately one-third [7] to one-half [5,6] of patients, a link between ALS and frontotemporal lobar degeneration (FTLD) has been made. Following the discovery of the CHMP2B gene mutation in both FTLD and ALS, some authors [8] have suggested that ALS and FTLD may be part of a pathological continuum and the term FTLD-MND has been introduced.

The diagnosis of ALS is primarily clinical and is confirmed by the demonstration of lower motor neuron loss on electromyography (EMG). It often takes months to establish the final clinical diagnosis of ALS and to merely express the suspicion of this fatal disease is a stressful event for the patient and his family, as well as for the physician. Therefore, any technique that supports or opposes the diagnosis of ALS or helps to improve the understanding of its origin and development is highly desirable.

Standard MR imaging of ALS patients has not revealed any unique imaging features apart from cortical atrophy, which is also characteristic to other neurodegenerative diseases. Some MR characteristics, such as increased signal intensity on FLAIR and T2-weighted images in the pyramidal tract (e.g., in the posterior limb of the internal capsule, PLIC) $[4,9,10]$, and T2-hypointensity in the motor cortex (precentral gyrus, "low signal rim") [3,10], have been attributed to ALS, but their diagnostic relevance is equivocal.

Quantitative MR imaging includes volumetric and morphometric techniques, measurements of proton $\mathrm{T} 1$ and $\mathrm{T} 2$ relaxation times ( $\mathrm{T} 1$ and $\mathrm{T} 2$ relaxometry), and quantitative aspects of water diffusion such as the apparent diffusion coefficient (ADC), mean diffusivity (MD), fractional anisotropy (FA) and proton MR spectroscopy (pMRS). Recent MRI studies [11,12] have shown the potential of quantitative MRI to improve the diagnostic process of ALS.

Thus, we elected to perform a cross-sectional imaging study in order to further evaluate the potential of quantitative MR techniques (voxel-based morphometry (VBM), T2relaxometry, FA, MD) in the diagnostic process of ALS and to further investigate the extent to which other brain regions are affected in ALS, especially the basal ganglia and nonmotor areas.

\section{Materials and methods}

\section{Participants}

A total of thirty-three subjects fulfilling the El-Escorial criteria [13] for clinically definite ALS were included in the study (18 men, 15 women; mean age $62.0 \pm 8.0 \mathrm{SD}$; disease duration 3-59 months); twenty subjects diagnosed with a limb-onset form of ALS, and thirteen diagnosed with a bulbar-onset form (Table 1). Disease severity was assessed with the ALS Functional Rating Scale (ALS-FRS), producing a score between 0 (worst) and 40 (best) [14]. As the total ALS-FRS score results from a mixture of both bulbar and limb origin impairment, we elected to split the scale to limb and bulbar components (Table 2) to allow the correlation of motor system morphology with clinical signs of motor disability. All subjects were further evaluated for the presence or absence of dementia by a series of neuropsychological tests [15]. Nineteen subjects exhibited a predominantly frontal-type cognitive impairment and behavioural disturbances consistent with FTLD, while twelve subjects were found to be cognitively normal. The results of neuropsychological examination were not available for two subjects.

Thirty healthy, age-matched volunteers (16 men, 14 women; mean age $59.7 \pm 10.0 \mathrm{SD}$ ) were also included in the study as a control group. All volunteers underwent an examination to exclude neurological disease. The study was approved by the local ethics committee and all participants provided signed informed consent.

\section{MR protocol}

Imaging was performed on a Siemens Symphony 1.5-Tesla System (Erlangen, Germany). Structural MR images were acquired using a T1-weighted magnetization-prepared rapid acquisition gradient echo (MP-RAGE) sequence (TR = $2140 \mathrm{~ms}, \mathrm{TE}=3.93 \mathrm{~ms}$, flip angle $=15$ degrees, $\mathrm{TI}=1100$ $\mathrm{ms}, 160$ axial slices, $1.65 \mathrm{~mm}$ slice thickness, $\mathrm{x}-\mathrm{y}$ resolution $0.9 \times 0.9 \mathrm{~mm}$, FOV $238 \mathrm{~mm}$ ) and a standard TSE T2-weighted sequence covering the entire brain and cerebellum. T2 relaxometry data were acquired by a single slice Carr-Purcell-Meiboom-Gill (CPMG) sequence ( 32 interecho times of $14.4,24.8,37.2$ to $396.8 \mathrm{~ms}$; TR $=3000 \mathrm{~ms}$; in-plane resolution $0.89 \times 0.89 \mathrm{~mm}$; slice thickness $4 \mathrm{~mm}$ ) in the region of the basal ganglia. Diffusion data were obtained using a Siemens work in progress (WIP) package with b-factors of 0,400 , and 1100 measured along 30 non-collinear directions $(\mathrm{TR}=7900 \mathrm{~ms}, \mathrm{TE}=101 \mathrm{~ms}, 70$ axial slices, $\mathrm{NEX}=2,1.25 \times 1.25 \times 2.5 \mathrm{~mm}$ ).

\section{MR data evaluation}

T2-weighted images covering the whole brain as well as raw data for quantitative analysis were evaluated to exclude other significant pathologies. VBM and T2 relaxometry studies were performed in thirty-one subjects (16 men, 15 women; mean age $61.6 \pm 8.0 \mathrm{SD}$ ). CPMG and VBM sequences were either incomplete or affected by artefacts in two subjects, and were excluded from VBM and T2 analysis. VBM analysis was performed using SPM2 (Wellcome Department 
Table 1 Demographic data for ALS patients

\begin{tabular}{|c|c|c|c|c|c|c|c|c|c|}
\hline Case no. & Age $^{a}$ & Sex & FRS & FRS-Limb & FRS-Bulbar & Form & Dementia & Duration $^{\mathrm{b}}$ & Scan \\
\hline \multicolumn{10}{|c|}{ ALS patient group } \\
\hline 1 & 66.1 & M & 31 & 18 & 13 & Limb & yes & 13 & $\mathrm{R}$ \\
\hline 2 & 58.5 & M & 31 & 15 & 16 & Limb & yes & 7 & $\mathrm{R}$ \\
\hline 3 & 67.5 & M & 33 & 24 & 9 & Bulbar & yes & 13 & $\mathrm{R}, \mathrm{D}$ \\
\hline 4 & 39.5 & M & 32 & 18 & 14 & Limb & yes & 54 & R, D \\
\hline 5 & 56.6 & M & 37 & 21 & 16 & Limb & no & 9 & $\mathrm{R}$ \\
\hline 6 & 76.2 & M & 36 & 24 & 12 & Limb & yes & 21 & $\mathrm{R}$ \\
\hline 7 & 67.1 & $\mathrm{~F}$ & 35 & 24 & 11 & Bulbar & yes & 24 & $\mathrm{R}$ \\
\hline 8 & 68.8 & M & 31 & 15 & 16 & Limb & yes & 13 & $\mathrm{R}$ \\
\hline 9 & 61.0 & $\mathrm{~F}$ & 24 & 9 & 15 & Limb & yes & 14 & $\mathrm{R}$ \\
\hline 10 & 61.9 & $\mathrm{~F}$ & 33 & 20 & 13 & Bulbar & yes & 8 & $\mathrm{R}$ \\
\hline 11 & 49.2 & M & 37 & 21 & 16 & Limb & no & 9 & $\mathrm{R}, \mathrm{D}$ \\
\hline 12 & 65.6 & M & 38 & 24 & 14 & Bulbar & yes & 4 & $\mathrm{R}$ \\
\hline 13 & 68.4 & $\mathrm{M}$ & 36 & 23 & 13 & Bulbar & yes & 7 & $\mathrm{R}, \mathrm{D}$ \\
\hline 14 & 65.5 & $\mathrm{~F}$ & 36 & 24 & 12 & Bulbar & yes & 17 & $\mathrm{R}$ \\
\hline 15 & 56.7 & $\mathrm{M}$ & 38 & 24 & 14 & Bulbar & no & 21 & $\mathrm{R}$ \\
\hline 16 & 61.1 & M & 30 & 15 & 15 & Limb & yes & 8 & $\mathrm{R}$ \\
\hline 17 & 63.3 & $\mathrm{~F}$ & 30 & 16 & 14 & Limb & yes & 12 & $\mathrm{R}$ \\
\hline 18 & 58.9 & M & 29 & 13 & 16 & Limb & yes & 11 & $\mathrm{R}$ \\
\hline 19 & 59.0 & $\mathrm{~F}$ & 39 & 24 & 15 & Bulbar & no & 8 & $\mathrm{R}$ \\
\hline 20 & 52.0 & $\mathrm{~F}$ & 34 & 21 & 13 & Bulbar & no & 9 & $\mathrm{R}$ \\
\hline 21 & 59.7 & $\mathrm{~F}$ & 37 & 24 & 13 & Bulbar & no & 12 & $\mathrm{R}$ \\
\hline 22 & 52.9 & $\mathrm{M}$ & 38 & 22 & 16 & Limb & no & 59 & $\mathrm{R}, \mathrm{D}$ \\
\hline 23 & 66.1 & $\mathrm{~F}$ & 32 & 16 & 16 & Limb & yes & 15 & $\mathrm{R}$ \\
\hline 24 & 64.8 & $\mathrm{M}$ & 40 & 24 & 16 & Limb & c & 5 & $\mathrm{R}$ \\
\hline 25 & 70.9 & $\mathrm{M}$ & 37 & 21 & 16 & Limb & yes & 14 & R, D \\
\hline 26 & 47.3 & $\mathrm{~F}$ & 32 & 19 & 13 & Limb & no & 8 & $\mathrm{R}$ \\
\hline 27 & 53.0 & M & 38 & 24 & 14 & Bulbar & no & 13 & $\mathrm{R}$ \\
\hline 28 & 78.6 & $\mathrm{~F}$ & 36 & 24 & 12 & Bulbar & yes & 14 & $\mathrm{R}, \mathrm{D}$ \\
\hline 29 & 73.3 & $\mathrm{~F}$ & 33 & 24 & 9 & Bulbar & no & 25 & $\mathrm{R}, \mathrm{D}$ \\
\hline 30 & 60.3 & $\mathrm{M}$ & 39 & 23 & 16 & Limb & no & 29 & $\mathrm{D}$ \\
\hline 31 & 65.2 & $\mathrm{~F}$ & 37 & 21 & 16 & Limb & yes & 25 & $\mathrm{R}, \mathrm{D}$ \\
\hline 32 & 71.6 & $\mathrm{M}$ & 38 & 22 & 16 & Limb & no & 41 & $\mathrm{D}$ \\
\hline 33 & 57.6 & M & 31 & 22 & 9 & Limb & $\mathrm{c}$ & 13 & $\mathrm{R}, \mathrm{D}$ \\
\hline
\end{tabular}

of Cognitive Neurology, London, UK) in the Matlab 6.5.1 environment (MathWorks, Natick, MA, USA) as previously described [16]. To suppress differences in image quality in comparison with the SPM T1-template, customized wholebrain $\mathrm{T} 1$ and tissue probability templates were used. The tissue probability templates were prepared by spatial normalization of the native T1 images to the SPM T1-template using both the patient and healthy volunteer groups, followed by segmentation into grey matter, white matter, and CSF compartments. The images were then blurred using an 8- $\mathrm{mm}$ full width at half maximum (FWHM) filter, averaged, and finally processed according to an optimized VBM protocol [16-18].
The original native T1 images were segmented, and the grey matter maps were normalized to the customized grey matter template. The normalization was processed by means of a 12-parameter affine transformation compensated for nonlinear shape differences $[19,20]$. The resulting normalization parameters were used to normalize the native T1 images before final segmentation. Simultaneously, inhomogeneities in the intensity of different grey and white matter regions were corrected for. All spatially normalized, segmented volumes were checked visually to identify any obvious tissue misclassification. Voxel values were then modulated by the Jacobian determinants derived from the normalization 
Table 2 ALS-FRS questionnaire divided into limb and bulbar components

\begin{tabular}{ll}
\hline Bulbar & Limb \\
\hline ALS-FRS components & \\
Speech & Handwriting \\
Salivation & Cutting food, handling utensils ${ }^{\mathrm{a}}$ \\
Swallowing & Dressing and hygiene \\
Dyspnoea & Turning in bed and adjusting bedclothes \\
& Walking \\
& Climbing stairs \\
\hline
\end{tabular}

ALS Amyotrophic lateral sclerosis, FRS Functional rating scale

${ }^{a}$ Patients without gastrostomy

process to provide local intensity correction due to regional volumetric signal expansion/reduction, thus preserving the volumes altered by nonlinear deformation [19]. Modulated grey and white matter maps were subsequently blurred isotropically using a 10- mm FWHM filter.

To verify the negative patient-control $t$-test, SPM8 with DARTEL [21] was used, correcting for age, sex, and intracranial volume. DARTEL is based on a fast diffeomorphic image registration algorithm using a "flow field" that is exponentiated to generate both forward and backward deformations. Segmentation is first performed followed by rigid transformation of the tissue class images, such that they are in as close alignment as possible with the tissue probability maps. Registration is then performed, which involves the simultaneous registration of grey and white matter. The mean of all images is used as an initial template, and the deformation each of the individual images to the template is computed. The template is then re-generated by applying the inverse of the deformation to each image and averaging. This procedure is repeated a number of times, and finally warped versions of the images are stored. The result of this process is better data coregistration in comparison with the optimized VBM approach using SPM2.

T2 relaxometry data were analysed using ImageJ viewer, placing circular regions of interest (ROIs) measuring twentyone square millimetres in the bilateral frontal white matter (FWM), frontal grey matter (FGM, cingulate gyrus) and PLIC. The basal ganglia (head of caudate nucleus, globus pallidus and putamen) were manually outlined bilaterally using freehand selection (Fig. 1). ROI placement was determined on the highest contrast $\mathrm{T} 2$ image, and the average signal data for each echo time were stored [22], generating 32 intensity values for each ROI. We performed nonlinear regression for each ROI using a monoexponential decay model for average signal intensity and interecho time with GraphPad Prism 4 (GraphPad Software Inc., La Jolla, CA, USA). PD values were individually calculated for echo time 0 in each ROI. T2 relaxation rate $(\mathrm{R} 2, \mathrm{R} 2=1 / \mathrm{T} 2)$ and proton density $(\mathrm{PD})$ sig-

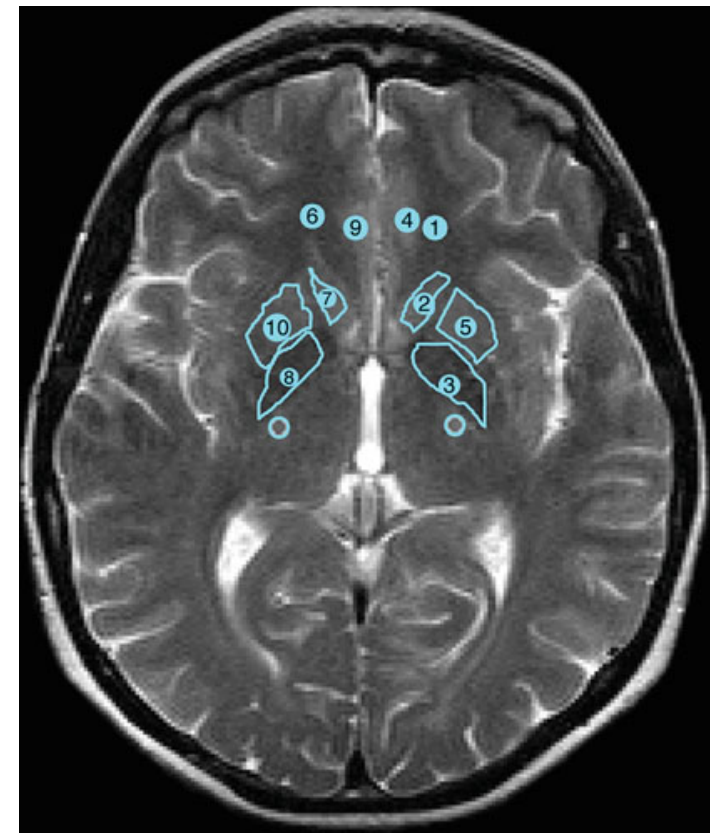

Fig. 1 Outlined regions of interest bilaterally: frontal white matter $(1,6)$, frontal grey matter $(4,9)$, head of caudate nucleus $(2,7)$, putamen $(5,10)$, globus pallidus $(3,8)$, and internal capsule (not numbered)

nal were statistically analysed using R-project version 2.4.1 [23] as described further.

Twelve subjects ( 9 men, 3 women; mean age $62.97 \pm$ 11.0 SD) and twelve healthy volunteers ( 9 men, 3 women; mean age $63.22 \pm 10.7 \mathrm{SD}$ ) were included in the DTI study. Diffusion weighted images were processed off-line in FSL (FMRIB Software Library [24]). After eddy-current and motion correction, brain extraction was performed using BET (brain extraction tool [25]) and DTIFit was then used to calculate the scalar invariants of the tensor. DTIFit generates $3 \mathrm{D}$ images at the same matrix size and resolution as the original diffusion images, including a raw T2-signal image with the same distortions as the diffusion weighted images. FA and MD were measured using ImageJ viewer, with circular ROIs measuring twenty-one square millimetres placed by a single observer (J.K.) in both the relaxometry and DTI studies. When analysing the CST region corresponding to the atrophy detected by VBM, ROIs were placed directly on the FA/MD maps (see Results). All other ROIs were placed directly on the T2 maps created by DTIFit, and then projected on the corresponding FA/MD map.

To validate the ROI approach, voxelwise statistical analysis of the FA data was carried out using TBSS (Tract-Based Spatial Statistics, [26]), part of FSL [24]. TBSS projects the FA data of all subjects onto a mean FA tract skeleton derived from the whole data set before applying voxelwise cross-subject statistics. For this analysis, only the upper slab covering the brain from the vertex to mesencephalon was used. The skeleton was built using an FA threshold $>0.3$. An unpaired 
$t$-test was then used to compare FA values in the patient and control group.

Statistical analysis

VBM data were statistically analysed by group comparison between patients and controls. Linear regression of local grey and white matter values in each voxel and the limb ALS-FRS score was performed. Montreal Neurological Institute (MNI) coordinates were used for the topographical description of results. Results were thresholded at a corrected cluster-level of $P<0.01$.

The R2 for each ROI was calculated as stated earlier. Normality was tested by the Shapiro test at a $P$-value threshold of $<0.05$. Linear regression analysis of R2 over age and ALS-FRS was performed and $P$-values were calculated. As normality of R2 in patients and controls was not confirmed by the Shapiro test in any region, a single-tailed Wilcoxon two-sample test was used for intergroup comparisons using an a priori hypothesis of decreased R2 in the patient group. Initially a $P$-value threshold of $<0.05$ was used, then Bonferonni correction was applied to decrease the alpha value threshold to 0.007 . The same methodology was used to evaluate the correlation between FA/MD, age, and clinical scale.

\section{Results}

VBM analysis of 31 ALS patients (confounding covariates: age, sex, intracranial volume as reported by SPM2) revealed a statistically significant correlation between decreased white matter intensity in the corona radiata and the limb component of ALS-FRS (Fig. 2a). The region of this correlation $(x=22, y=-27, z=51 ; T=3.48 ; P<0.01$ clusterlevel corrected) is shown in Fig. 2b. This region was further investigated by diffusion analysis in a subgroup of twelve patients, and no correlation between clinical scale/age and FA/MD was observed.

An unpaired $t$-test corrected for age, sex and brain volume was used for comparison of both white and grey matter intensities. In SPM2 no significant correlations were observed. SPM8 (using DARTEL, $P<0.05$ family-wise error (FWE) corrected) revealed decreased white matter intensity in the corona radiata bilaterally (Fig. 3). One of the blobs is very near the correlation site in the precentral gyrus (position $x=$ 28, $y=-23, z=54 ; T=5.89 ; P<0.05$ FWE). Using a threshold of $P<0.0001$ uncorrected, the site of correlation with the limb component of FRS is included in the resulting statistical map.

We detected significantly decreased $\mathrm{R} 2$ in the head of the caudate nuclei (left $P<0.005$, right $P<0.05$, uncorrected) in ALS patients in comparison with healthy volunteers (Table 3). No dependence of R2 on the duration of
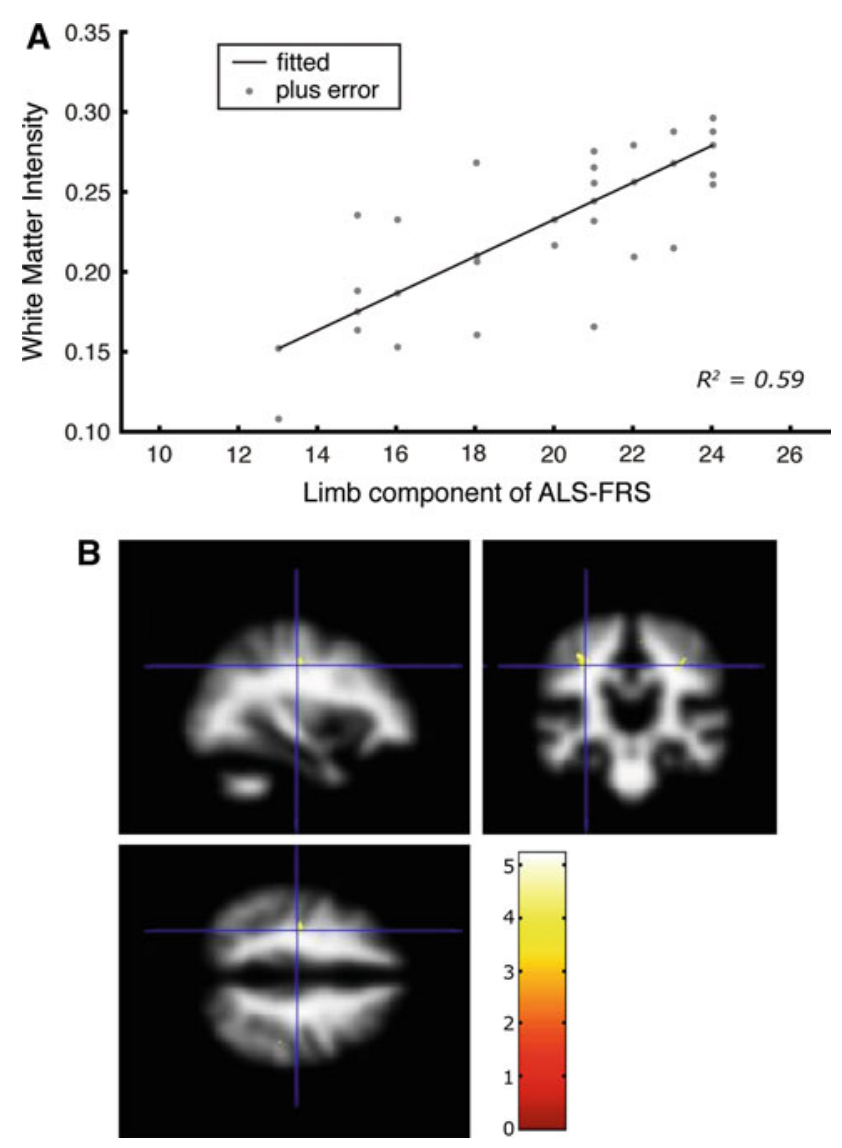

Fig. 2 a Voxel-based morphometry (VBM) fitted white matter intensity in correlation with the limb component of the ALS Functional Rating Scale (ALS-FRS). b Region of statistically significant correlation between white matter atrophy and the limb component of ALS Functional Rating Scale (ALS-FRS). T-value represented by colour gradient

disease was observed, with a similar increase in R2 observed across the range of disease duration present in our crosssectional study. Lower R2 was also present in the deep FWM in ALS patients in comparison with controls (left and right $P<0.005)$. No statistically significant differences in the head of the caudate nuclei or FWM were found between the subgroups of ALS patients with/without cognitive deficit. After Bonferroni correction (corrected alpha value 0.007), the statistically significant differences between patients and controls in the head of the left caudate nucleus and bilateral FWM remained. R2 in the FWM correlated with age in both patients and controls (Fig. 4). Changes of R2 in the FGM did not exceed the statistical threshold.

No statistically significant changes were detected in the PLIC, a region suspected of signal intensity changes in ALS. Changes in $\mathrm{R} 2$ in this region did not exceed the statistical threshold nor did any differences in PD (Figs. 5 and 6). T2 hyperintensity in the PLIC was qualitatively appreciated at least unilaterally in all patients and controls (Fig. 7).

Using an ROI-based approach, no correlation between clinical scale/age and FA/MD was observed in the PLIC. 

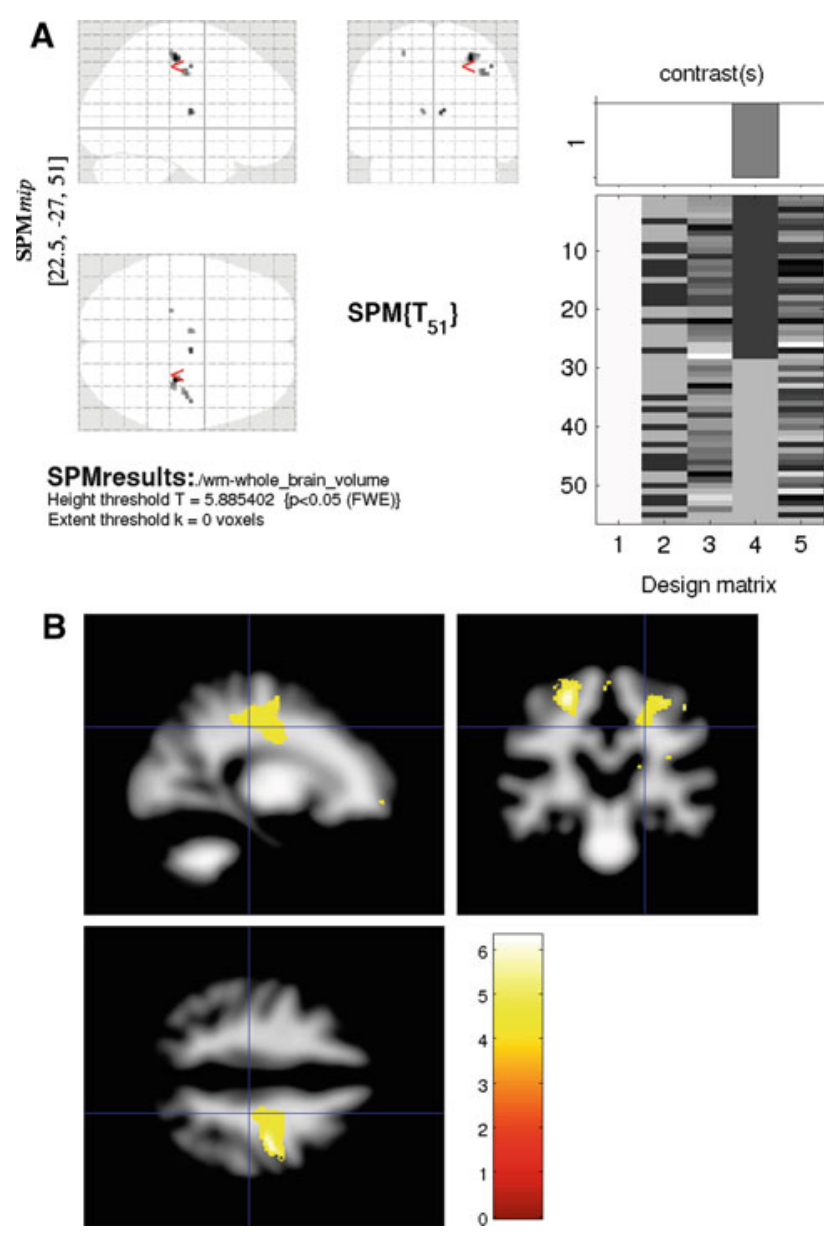

Fig. 3 Voxel-based morphometry using DARTEL (fast diffeomorphic image registration algorithm). Difference between white matter intensity in patients and controls. a $P<0.05$ family-wise error corrected, crosshair indicates correlation site with the limb component of ALS Functional Rating Scale (ALS-FRS) (for details see Fig. 2). b $P<$ 0.0001 uncorrected

Furthermore, no significant differences in FA were detected between patients and healthy volunteers in the PLIC or FWM. We therefore elected to perform TBSS on the upper slab of diffusion data. No correlation with FRS was found, but a significant difference $(P<0.05$ corrected) between the patient group and control group was present in the corona radiata (Fig. 5).

\section{Discussion}

We found a statistically significant correlation between decreased white matter intensity in the corona radiata and ALS-FRS (Fig. 2) in ALS patients. White matter analysis using DARTEL further revealed lower white matter intensity in ALS patients compared to healthy controls in the same anatomical location. Our findings suggest that as in other neurodegenerative diseases, FWM atrophy may be a surrogate
Table 3 T2 relaxation time in milliseconds for patients and controls

\begin{tabular}{lrrrrr}
\hline Region & Patients & SD & Controls & SD & $P$-value \\
\hline ALS relaxometry results & & & & & \\
Head CN, L* & 99.7 & 6.4 & 95.6 & 3.4 & 0.0018 \\
Head CN, R & 99.0 & 7.1 & 96.6 & 4.5 & 0.0232 \\
FWM, L* & 88.8 & 7.8 & 84.0 & 4.9 & 0.0019 \\
FWM, R* & 88.2 & 6.3 & 84.4 & 4.5 & 0.0044 \\
GP, L & 72.0 & 5.4 & 73.0 & 5.7 & \\
GP, R & 72.2 & 5.9 & 73.7 & 6.6 & \\
CG, L & 107.9 & 8.6 & 107.1 & 4.0 & \\
CG, R & 105.2 & 10.6 & 107.6 & 5.4 & \\
Putamen, L & 88.8 & 5.9 & 87.8 & 5.6 & \\
Putamen, R & 88.4 & 6.9 & 88.7 & 6.3 & \\
CST, L & 107.3 & 7.0 & 106.5 & 4.4 & \\
CST, R & 107.6 & 6.6 & 108.0 & 5.2 &
\end{tabular}

$A L S$ Amyotrophic lateral sclerosis, $S D$ Standard deviation, $L$ Left, $R$ Right, $C N$ Caudate Nucleus, $F W M$ Frontal white matter, GP Globus pallidus, $C G$ Cingulate gyrus, CST Corticospinal tract

Significant difference as reported by single-tailed Wilcoxon test marked as $* P<0.05$ after Bonferroni correction

marker of ALS pathology on MRI rather than cortical grey matter involvement. A similar observation was made by Sage et al. [27], where a correlation between the entire ALS-FRS scale and FA in the same region was found. The demographic data of our study and the study of Sage et al. are similar in the number of patients included as well as in the post-processing algorithm.

VBM in ALS has been performed in various studies, often with controversial results. For instance, Mezzapesa et al. [28] described signs of frontotemporal atrophy in ALS patients compared to controls. Kassubek et al. reported similar results [29]. Agosta et al. [30] found lower grey matter density in the superior temporal gyri bilaterally, as well as in the right precentral and left inferior frontal gyri. Chang et al. [31] reported atrophy of the medial frontal gyrus. These studies differ in the number of patients included and the methodology used. This may, at least in part, explain the discrepancies in their results.

The use of an "optimized" VBM protocol as suggested by SPM2 and used by default in SPM5 resulted in falsenegative results in the group comparison, contrary to DARTEL implemented in SPM8. These differences are not widely appreciated and should be taken in account when planning or interpreting VBM studies.

To the best of our knowledge, this is the first published account of changes in $\mathrm{T} 2$ relaxation rate in the head of caudate nucleus in ALS. Atrophy of the head of the left caudate was found in a subgroup of rapidly progressing ALS patients in another study [32]. These observations support the hypothesis that subcortical grey matter areas may be significantly involved in the pathogenesis of ALS. Significant changes of 
Fig. 4 Correlation between T2 relaxation rate (inverse seconds) and age (years)

Fig. 5 Tract-based spatial statistics (TBSS) comparison of FA between patients and controls $(t$-test patient $<$ control, $P<0.05$ corrected, skeleton threshold $0.3)$
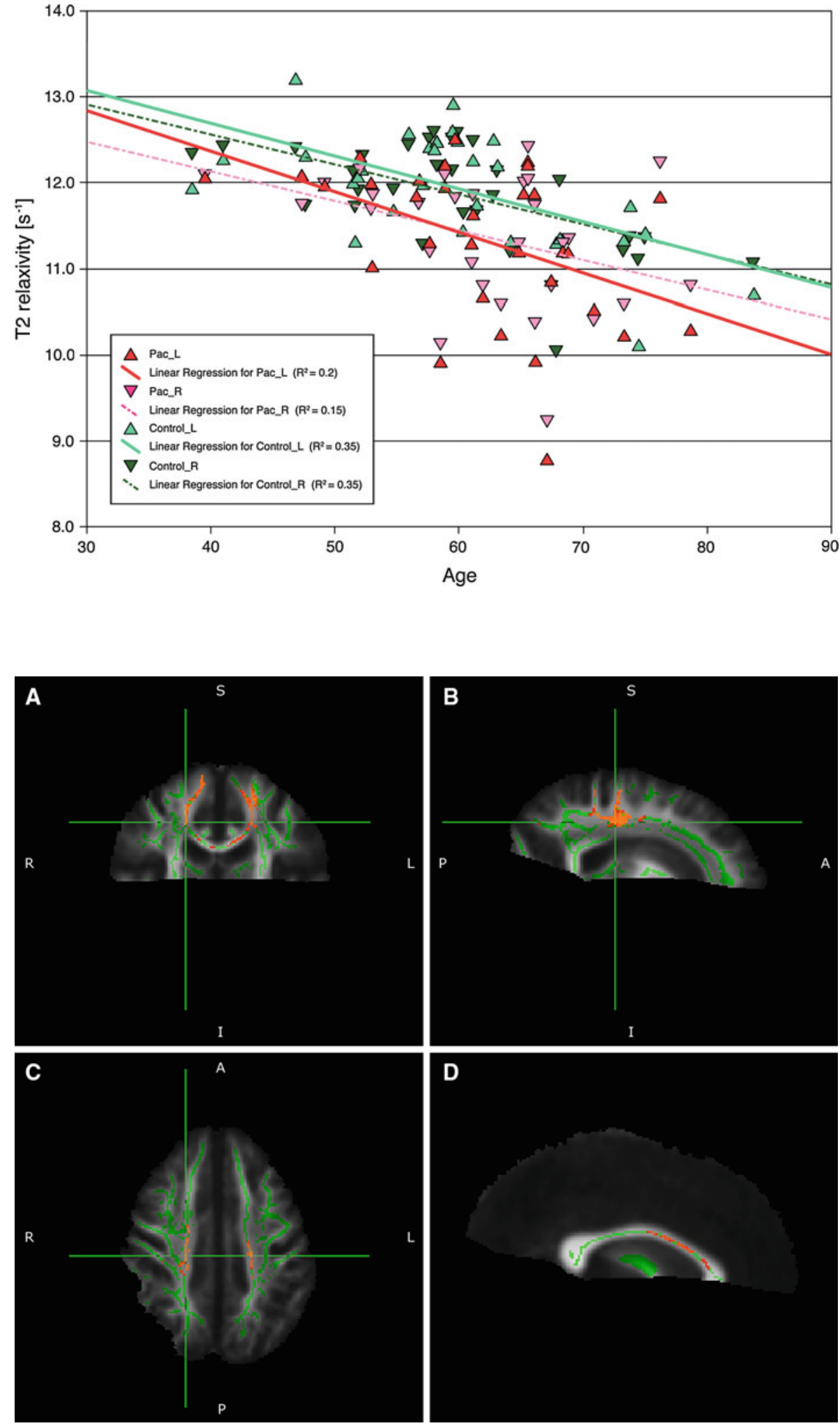

$\mathrm{T} 2$ relaxation rate in this region are of further importance as the caudate nucleus has been used as an internal reference in qualitative and quantitative studies of ALS patients
[33-35]. It is interesting to note that the relaxation rate changes detected in this region were not dependent on disease duration. This suggests that decreased R2 is already present 
Fig. 6 Comparison of R2 (inverse seconds) between patients and controls in various brain regions. Bars represent the mean and error bars represent standard deviation. $L$ left, $R$ right, $C N$ head of caudate nucleus, $F W M$ frontal white matter, $G P$ globus pallidus, $C G$ cingulate gyrus, $P U T$ putamen

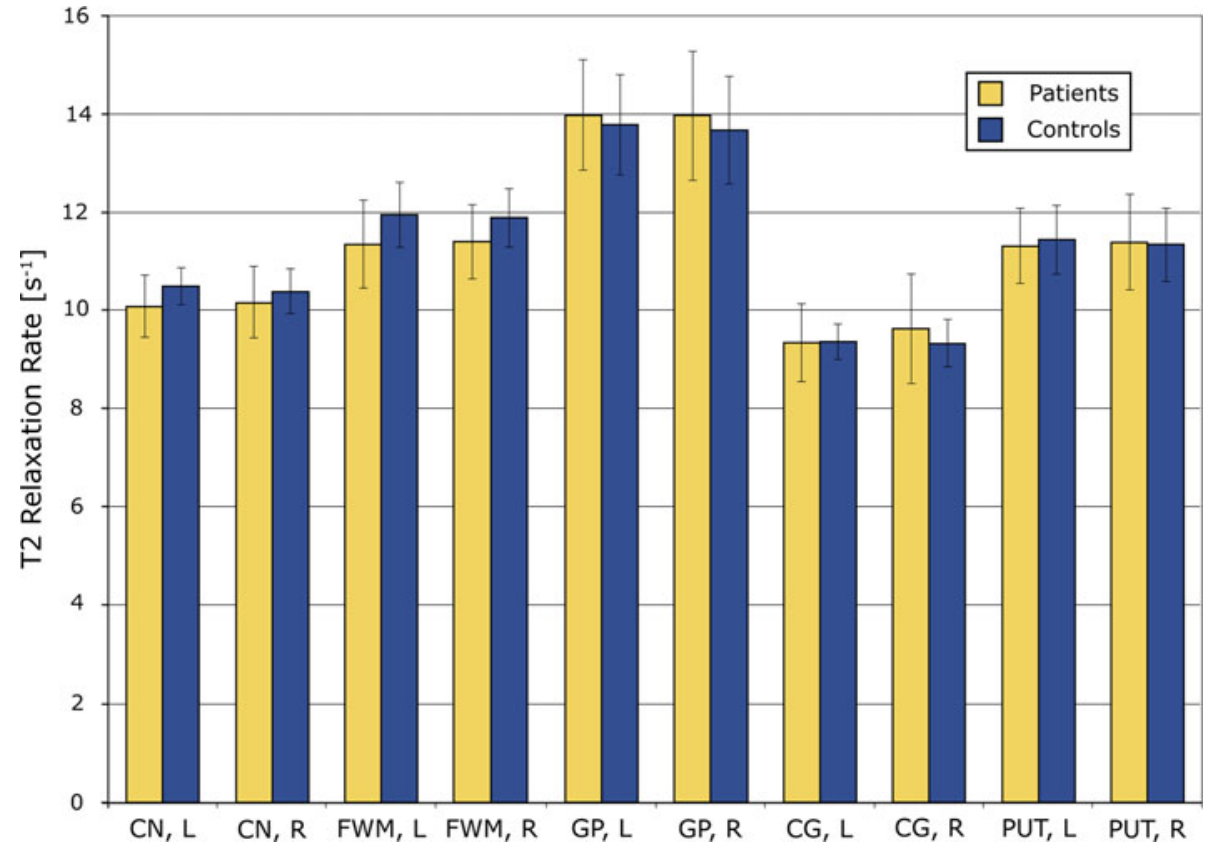

in the earlier stages of the disease and could therefore serve as an early marker of disease activity. Future investigation should focus on quantifying changes in head of the caudate nuclei in a large group patients in the earliest stage of ALS.

Significant changes in the $\mathrm{T} 2$ relaxation rate of the caudate nucleus are not surprising when considering that ALS is not limited to the motor system and that relaxometric changes in the basal ganglia have been observed in other neurodegenerative diseases [36]. T2 contrast in the deep grey matter nuclei is predominantly determined by tissue iron in metalloproteins $[37,38]$. A decrease in the metalloprotein-bound iron ("safe" iron) results in a relative decrease in $\mathrm{R} 2$, and more iron in the low molecular forms ("toxic" iron) is available. We propose that the significant $\mathrm{T} 2$ relaxation rate changes in the left caudate nucleus observed in our study are related to iron deposition in this region. The observed changes cannot be explained by a change in water content as the proton density did not differ between patients and controls.

We also detected significantly decreased R2 in the bilateral FWM of ALS patients compared to controls. Therefore, we recommend that FWM (as well as head of caudate nuclei, see above) should not be used as an internal reference for the assessment of increased signal intensity in various brain regions in ALS $[34,35]$. The T2 relaxation rate in the FWM was also found to correlate with age in both patients and controls. Bartzokis et al. demonstrated the age dependence of R2 in the FWM at both 0.5 and 1.5 Tesla field strengths in healthy subjects [39]. A trend towards a steeper decrease of T2 relaxation rate with age in our group of ALS patients was observed, but the difference did not reach the statistical threshold. As age-related changes in the FWM were detected in both patients and controls, this may indicate that decreased
R2 in the FWM is age-dependent and that ALS does not influence this correlation significantly.

No statistically significant changes were detected in the PLIC, a region suspected of signal intensity changes in ALS. T2 hyperintensity in this area, labelled as CI3 in histological studies, was observed in nearly all subjects. A confocal microscopy study by Axer [40] revealed that fibres of the corticospinal tract (running superoinferior) cross fibres of the upper thalamic peduncle (running anteroposterior) in this region. In section, a pale area in the most posterior part of internal capsule was more difficult to discern and was less stained by silver than by myelin stain in ALS subjects. This area included thinner axons ( $>0.8 \mu \mathrm{m})$, surrounded by "free space". The posterior aspect of the area was clearly delimited, and the anterior aspect disappeared gradually. In ALS patients, the loss of thin myelinated fibres in this area has been proven [41]. Even though these findings would suggest decreased R2 in this area, our study quantitatively confirms earlier statements $[9,10]$ that the relatively hyperintense signal in this part of the internal capsule is a physiological variant and not disease related as was believed [4,42].

TBSS revealed decreased FA in the area corresponding to the site of atrophy detected by VBM in ALS patients compared to healthy volunteers. These changes were observed in the corona radiata, and no changes were detected in the lower parts of the pyramidal tract. No changes in FA in the corticospinal tract were found using an ROI-based approach. This can be at least partially explained by the process of averaging the data on the skeleton in a voxel-wise manner, which, in contrast to an ROI-based approach, is not influenced by selection size or a priori knowledge. Moreover, an ROI positioned close to fine white fibres is more prone to noise. 


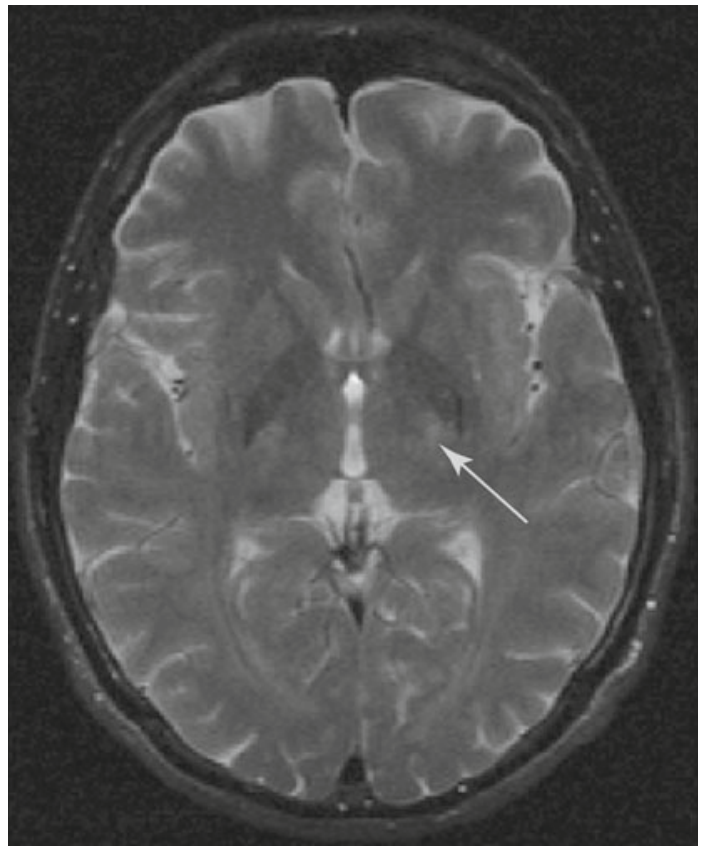

Fig. 7 T2 hyperintensity in the posterior limb of the internal capsule (arrow) in a healthy volunteer

No changes in the FWM were detected by means of TBSS or ROI-based methods. Decreased FA in the callosal body is in agreement with current knowledge of fronto-temporal lobar degeneration. Therefore, future research should focus more on fine fibres. Changes in R2 and not FA in this region can be explained by demyelinating changes in the white matter that precede changes in anisotropy. Thus, changes in $\mathrm{T} 2$ relaxation rate may precede decreased diffusion anisotropy.

Although a direct comparison of the quantitative MR methods used in our study is not fair as the sequences used differ in slice thickness (ranging from 1.65 to $4 \mathrm{~mm}$ ) and inplane resolution, thereby exhibiting various signal-to-noise ratios, our data suggest that a combination of several quantitative methods may be the key to improving the role of MRI in the diagnostic process.

\section{Conclusions}

We have demonstrated that pathological changes in ALS are widespread and not limited to only the motor system. R2 in the head of the left caudate and bilateral FWM differs between ALS patients and controls, and these regions cannot be used as an internal reference of "healthy tissue" as in previous studies. A significant correlation was also observed between white matter intensity in the corona radiata and the limb component of ALS-FRS, suggesting that FWM atrophy rather than cortical grey matter involvement may be a surrogate marker of ALS pathology on MRI. TBSS further revealed decreased FA in ALS patients in the corona radiata and callosal body, reflecting pathological changes in the motor pathway and white matter of the frontal and temporal lobes. These findings indicate regions that may be important in the future diagnostic process of ALS.

Although T2 hyperintensity in the PLIC has been considered a marker of ALS, our quantitative analysis of the pyramidal tract passing through the PLIC did not reveal any significant differences between ALS patients and healthy volunteers. This indicates that T2 hyperintensity in the PLIC should no longer be considered a marker of ALS on MRI.

Acknowledgments Supported from grants IGA MZ ČR, NS96544/2008, NR8491-3/2007, NR8937-4/2006 and research programs MŠM 0021620849 and MŠM 0021620816.

Open Access This article is distributed under the terms of the Creative Commons Attribution Noncommercial License which permits any noncommercial use, distribution, and reproduction in any medium, provided the original author(s) and source are credited.

\section{References}

1. Adams V, Victor M (1993) Principles of neurology. 5th edn. McGraw-Hill Inc, New York

2. Atlas S (2001) Magnetic resonance imaging of the brain and spine. 3rd edn. Lippincott Williams and Wilkins, Philadelphia

3. Oba H, Araki T, Ohtomo K, Monzawa S, Uchiyama G, Koizumi K, Nogata Y, Kachi K, Shiozawa Z, Kobayashi M (1993) Amyotrophic lateral sclerosis: $\mathrm{T} 2$ shortening in motor cortex at MR imaging. Radiology 189(3):843-846

4. Hofmann E, Ochs G, Pelzl A, Warmuth-Metz M (1998) The corticospinal tract in amyotrophic lateral sclerosis: an MRI study. Neuroradiology 40(2):71-75

5. Phukan J, Pender NP, Hardiman O (2007) Cognitive impairment in amyotrophic lateral sclerosis. Lancet Neurol 6(11):994-1003

6. Murphy J, Henry R, Lomen-Hoerth C (2007) Establishing subtypes of the continuum of frontal lobe impairment in amyotrophic lateral sclerosis. Arch Neurol 64(3):330-334

7. Rippon GA, Scarmeas N, Gordon PH, Murphy PL, Albert SM, Mitsumoto H, Marder K, Rowland LP, Stern Y (2006) An observational study of cognitive impairment in amyotrophic lateral sclerosis. Arch Neurol 63(3):345-352

8. Lomen-Hoerth C, Murphy J, Langmore S, Kramer JH, Olney RK, Miller B (2003) Are amyotrophic lateral sclerosis patients cognitively normal. Neurology 60(7):1094-1097

9. Goodin DS, Rowley HA, Olney RK (1988) Magnetic resonance imaging in amyotrophic lateral sclerosis. Ann Neurol 23(4):418420

10. Cheung G, Gawel MJ, Cooper PW, Farb RI, Ang LC, Gawal MJ (1995) Amyotrophic lateral sclerosis: correlation of clinical and MR imaging findings. Radiology 194(1):263-270

11. Sage CA, Van Hecke W, Peeters R, Sijbers J, Robberecht W, Parizel P, Marchal G, Leemans A, Sunaert S (2009) Quantitative diffusion tensor imaging in amyotrophic lateral sclerosis: revisited. Hum Brain Mapp 30(11):3657-3675

12. Cosottini M, Giannelli M, Siciliano G, Lazzarotti G, Michelassi MC, Del Corona A, Bartolozzi C, Murri L (2005) Diffusion-tensor MR imaging of corticospinal tract in amyotrophic lateral sclerosis and progressive muscular atrophy. Radiology 237(1):258-264 
13. Brooks BR (1994) El escorial world federation of neurology criteria for the diagnosis of amyotrophic lateral sclerosis, subcommittee on Motor Neuron Diseases/Amyotrophic Lateral Sclerosis of the World Federation of Neurology Research Group on Neuromuscular Diseases and the El Escorial "Clinical limits of amyotrophic lateral sclerosis" workshop contributors. J Neurol Sci 144 (Suppl):96-107

14. The ALS CNTF treatment study (ACTS) phase I-II Study Group (1996) The amyotrophic lateral sclerosis functional rating scale. Assessment of activities of daily living in patients with amyotrophic lateral sclerosis. Arch Neurol 53(2):141-147

15. Rusina R, Ridzoň $P$, Kulišt’ák $P$, Keller $O$, Bartoš A, Buncová M, Fialová L, Koukolík F, Matěj R (2010) Relationship between ALS and the degree of cognitive impairment, markers of neurodegeneration and predictors for poor outcome. A prospective study. Eur J Neurol 17(1):23-30

16. Jech R, Klempír J, Vymazal J, Židovská J, Klempîrová O, Růžička E, Roth J (2007) Variation of selective gray and white matter atrophy in Huntington's disease. Movement Disorders 22(12):17831789

17. Good CD, Johnsrude IS, Ashburner J, Henson RN, Friston KJ, Frackowiak RS (2001) A voxel-based morphometric study of ageing in 465 normal adult human brains. Neuroimage 14(1 Pt 1): $21-36$

18. Good CD, Johnsrude IS, Ashburner J, Henson RN, Friston KJ, Frackowiak RS (2001) Cerebral asymmetry and the effects of sex and handedness on brain structure: a voxel-based morphometric analysis of 465 normal adult human brains. Neuroimage 14: $685-700$

19. Ashburner J, Friston KJ (2000) Voxel-based morphometry-the methods. Neuroimage 11(6 Pt 1):805-821

20. Ashburner J, Friston KJ (2001) Why voxel-based morphometry should be used. Neuroimage 14:1238-1243

21. Ashburner $\mathbf{J}$ (2007) A fast diffeomorphic image registration algorithm. NeuroImage 38(1):95-113

22. Rasband W (1997-2008) ImageJ. http://rsb.info.nih.gov/ij/

23. R Development Core Team (2009) R: a language and environment for statistical computing. R Foundation for Statistical Computing, Vienna, Austria, URL http://www.R-project.org, ISBN 3-90005107-0

24. Smith SM, Jenkinson M, Woolrich MW, Beckmann CF, Behrens TEJ, Johansen-Berg H, Bannister PR, De Luca M, Drobnjak I, Flitney DE, Niazy RK, Saunders J, Vickers J, Zhang Y, De Stefano N, Brady JM, Matthews PM (2004) Advances in functional and structural MR image analysis and implementation as FSL. Neuroimage 23(1):S208-S219

25. Smith SM (2002) Fast robust automated brain extraction. Hum Brain Mapp 17(3):143-155

26. Smith SM, Jenkinson M, Johansen-Berg H, Rueckert D, Nichols TE, Mackay CE, Watkins KE, Ciccarelli O, Cader MZ, Matthews PM, Behrens TEJ (2006) Tract-based spatial statistics: Voxelwise analysis of multi-subject diffusion data. NeuroImage 31:14871505

27. Sage CA, Peeters RR, Görner A, Robberecht W, Sunaert S (2007) Quantitative diffusion tensor imaging in amyotrophic lateral sclerosis. Neuroimage 34(2):486-499

28. Mezzapesa DM, Ceccarelli A, Dicuonzo F, Carella A, De Caro MR, Lopez M, Samarelli V, Livrea P, Simone IL (2007) Wholebrain and regional brain atrophy in amyotrophic lateral sclerosis. AJNR Am J Neuroradiol 28(2):255-259
29. Kassubek J, Unrath A, Huppertz HJ, Lule D, Ethofer T, Sperfeld $\mathrm{AD}$, Ludolph AC (2005) Global brain atrophy and corticospinal tract alterations in ALS, as investigated by voxel-based morphometry of 3-D MRI, Amyotrophic lateral sclerosis and other motor neuron disorders: official publication of the World Federation of Neurology. Res Group Motor Neuron Diseases 6(4):213-220

30. Agosta F, Pagani E, Rocca MA, Caputo D, Perini M, Salvi F, Prelle A, Filippi M (2007) Voxel-based morphometry study of brain volumetry and diffusivity in amyotrophic lateral sclerosis patients with mild disability. Hum Brain Mapp 28(12):1430-1438

31. Chang JL, Lomen-Hoerth C, Murphy J, Henry RG, Kramer JH, Miller BL, Gorno-Tempini ML (2005) A voxel-based morphometry study of patterns of brain atrophy in ALS and ALS/FTLD. Neurology 65(1):75-80

32. Agosta F, Gorno-Tempini ML, Pagani E, Sala S, Caputo D, Perini M, Bartolomei I, Fruguglietti ME, Filippi M (2009) Longitudinal assessment of grey matter contraction in amyotrophic lateral sclerosis: a tensor based morphometry study. Amyotroph Lateral Scler 10(3): 168-174

33. Ngai S, Tang YM, Du L, Stuckey S (2007) Hyperintensity of the precentral gyral subcortical white matter and hypointensity of the precentral gyrus on fluid-attenuated inversion recovery: variation with age and implications for the diagnosis of amyotrophic lateral sclerosis. AJNR Am J Neuroradiol 28(2):250-254

34. Hecht MJ, Fellner F, Fellner C, Hilz MJ, Heuss D, Neundörfer B (2001) MRI-FLAIR images of the head show corticospinal tract alterations in ALS patients more frequently than T2-, T1- and proton-density-weighted images. J Neurol Sci 186(1-2):37-44

35. Hecht MJ, Fellner F, Fellner C, Hilz MJ, Neundörfer B, Heuss D (2002) Hyperintense and hypointense MRI signals of the precentral gyrus and corticospinal tract in ALS: a follow-up examination including FLAIR images. J Neurol Sci 199(1-2):59-65

36. Bartzokis G, Tishler TA (2000) MRI evaluation of basal ganglia ferritin iron and neurotoxicity in Alzheimer's and Huntingon's disease. Cell Mol Biol 46(4):821-833

37. Vymazal J, Hájek M, Patronas N, Giedd JN, Bulte JW, Baumgarner C, Tran V, Brooks RA (1995) The quantitative relation between T1-weighted and T2-weighted MRI of normal gray matter and iron concentration. J Magn Reson Imaging 5(5):554-560

38. Vymazal J, Brooks RA, Patronas N, Hájek M, Bulte JW, Di Chiro $\mathrm{G}$ (1995) Magnetic resonance imaging of brain iron in health and disease. J Neurol Sci 134(Suppl):19-26

39. Bartzokis G, Mintz J, Sultzer D, Marx P, Herzberg JS, Phelan CK, Marder SR (1994) In vivo MR evaluation of age-related increases in brain iron. AJNR Am J Neuroradiol 15(6):1129-1138

40. Axer H, Keyserlingk DG (2000) Mapping of fiber orientation in human internal capsule by means of polarized light and confocal scanning laser microscopy. J Neurosci Methods 94(2):165-175

41. Hirayama K, Tsubaki T, Toyokura Y, Okinaka S (1962) The representation of the pyramidal tract in the internal capsule and basis pedunculi. A study based on three cases of amyotrophic lateral sclerosis. Neurology 12:337-342

42. Yagishita A, Nakano I, Oda M, Hirano A (1994) Location of the corticospinal tract in the internal capsule at MR imaging. Radiology 191(2):455-460 